Mens

Revue d'histoire intellectuelle et culturelle

mens

\title{
Un valentin au grand Maurice - Léon Bloy, Olivar Asselin et Robert LaPalme : essai sur un problème de transfert culturel dans l'histoire de la caricature au Québec
}

\section{Dominic Hardy}

Volume 12, numéro 2, printemps 2012

Enjeux et modalités des transferts culturels dans la vie artistique canadienne-française de la première moitié $d u \mathrm{XX}^{\mathrm{e}}$ siècle

URI : https://id.erudit.org/iderudit/1013875ar

DOI : https://doi.org/10.7202/1013875ar

Aller au sommaire du numéro

Éditeur(s)

Centre de recherche en civilisation canadienne-française

ISSN

1492-8647 (imprimé)

1927-9299 (numérique)

Découvrir la revue

Citer cet article

Hardy, D. (2012). Un valentin au grand Maurice — Léon Bloy, Olivar Asselin et Robert LaPalme : essai sur un problème de transfert culturel dans l'histoire de la caricature au Québec. Mens, 12(2), 103-143.

https://doi.org/10.7202/1013875ar
Résumé de l'article

En partant d'une caricature remarquablement violente de Maurice Duplessis, dessinée par Robert LaPalme et publiée dans l'hebdomadaire Vrai en février 1958, la présente étude fait état de certains défis que posent l'analyse et l'interprétation de l'image satirique dans l'histoire et, plus généralement, dans l'histoire de la culture littéraire et artistique au Québec. Les recherches sur la carrière de LaPalme montrent l'importance qu'il faut accorder à son milieu intellectuel et idéologique, de par son association avec quelques-uns des plus influents journalistes-éditeurs de son temps. Or une figure surprenante émerge de ces recherches : celle de l'écrivain français Léon Bloy. Les traces de son influence semblent nombreuses mais demeurent difficiles à dégager. Nous croyons pourtant qu'il s'agit bel et bien d'un cas de transfert culturel qui s'opère depuis la littérature polémique bloyenne du tournant $\mathrm{du} \mathrm{xx}^{\mathrm{e}}$ siècle vers cette autre littérature polémique, souvent illustrée, qui est pratiquée au Québec dans les années 1930-1950 par les Olivar Asselin, Claude-Henri Grignon, André Laurendeau et, bien entendu, Robert LaPalme. 


\title{
Un valentin au grand Maurice Léon Bloy, Olivar Asselin et Robert LaPalme : essai sur un problème de transfert culturel dans l'histoire de la caricature au Québec ${ }^{1}$
}

\author{
Dominic Hardy \\ Département d'histoire de l'art \\ Université du Québec à Montréal
}

\section{Résumé}

En partant d'une caricature remarquablement violente de Maurice Duplessis, dessinée par Robert LaPalme et publiée dans l'hebdomadaire Vrai en février 1958, la présente étude fait état de certains défis que posent l'analyse et l'interprétation de l'image satirique dans l'histoire et, plus généralement, dans l'histoire de la culture littéraire et artistique au Québec. Les recherches sur la carrière de LaPalme montrent l'importance qu'il faut accorder à son milieu intellectuel et idéologique, de par son association avec quelques-uns des plus influents journalistes-éditeurs de son temps. Or une figure surprenante émerge de ces recherches : celle de l'écrivain français Léon Bloy.

\footnotetext{
${ }^{1}$ Nous tenons à exprimer notre vive reconnaissance à Stéphanie Danaux et Nova Doyon pour les nombreux conseils et commentaires qu'elles nous ont généreusement offerts au cours de la rédaction de ce texte, qui développe un thème introduit dans nos recherches doctorales consacrées à l'étude des contextes de la caricature antiduplessiste de Robert LaPalme. Voir Dominic Hardy, A Metropolitan Line: Robert LaPalme, Caricature and Power in the Age of Duplessis, thèse de doctorat, Montréal, Université Concordia, 2006.
} 
Les traces de son influence semblent nombreuses mais demeurent difficiles à dégager. Nous croyons pourtant qu'il s'agit bel et bien d'un cas de transfert culturel qui s'opère depuis la littérature polémique bloyenne du tournant $\mathrm{du} \mathrm{xx}^{\mathrm{e}}$ siècle vers cette autre littérature polémique, souvent illustrée, qui est pratiquée au Québec dans les années 1930-1950 par les Olivar Asselin, Claude-Henri Grignon, André Laurendeau et, bien entendu, Robert LaPalme.

\section{Abstract}

Starting with the remarkably violent caricature of Maurice Duplessis drawn by Robert LaPalme and published in the weekly Vrai in February 1958, this paper articulates certain challenges, at the levels of analysis and interpretation, that have to be taken into account in thinking about the satiric image in the history, and in the history of literary and artistic culture more generally, of Québec. Research on the career of LaPalme shows the importance of his intellectual and ideological milieux, notably through his association with some of the most influential journalists and editors of his time. A surprising figure emerges through this research: that of the French writer Léon Bloy, the traces of whose influence are many, although difficult to situate in terms of the transfer from his own early $X X^{\text {th }}$ century polemic literature to this other, often illustrated polemic literature that is marked by figures such as Olivar Asselin, Claude-Henri Grignon, André Laurendeau and, of course, Robert LaPalme.

\section{Arrêt sur image, traces fugitives : termes d'une enquête}

De toute la production satirique que consacra l'artiste Robert LaPalme (1908-1997) au premier ministre Maurice Duplessis (18901959) entre 1943 et 1959, une image dépasse toutes les autres par sa volonté de mettre à nu le Chef et d'en faire un vecteur visible de violence. Il s'agit de la caricature publiée à la une de l'édition du 22 février 1958 de l'hebdomadaire Vrai², qu'avait fondé Jacques Hébert

2 Robert LaPalme, «Un valentin au grand Maurice », Vrai, vol. 4, n 25 (22 février 1958), p. 1. 
(1923-2007) en 1954 pour soutenir les efforts de la nouvelle formation municipale, la Ligue d'action civique (LAC), dirigée par Jean Drapeau. La manchette nous donne la mesure de l'opposition du journal et de la LAC au gouvernement de Duplessis. Ainsi, la phrase "La démocratie violée à Québec ", en plus d'actualiser une virulence qu'on n'avait pas vue au Québec depuis les jours de $L a$ Nation (1936-1939), donne le ton à la page. Elle nous prépare aussi au dessin de Robert LaPalme, Un valentin au grand Maurice (Figure 1).

Ce grand Maurice s' incline tout en se tordant vers nous. Il émerge d'un fond tout à fait noir (une grande noirceur); son corps mis à nu s'étend aux limites de l'image. Ses bras et ses jambes sont rendus selon la sinuosité caractéristique du style de LaPalme depuis 1943, année de son entrée au Canada où il entame sa longue lutte contre Duplessis avant de passer au Devoir en 1952, dont il sera le premier caricaturiste attitré jusqu'en janvier $1959^{3}$. Une palette assez réduite de signes est utilisée pour préciser ce corps stylisé. La peau du tronc, de la cuisse et du tibia est légèrement poilue. Un point, ou plutôt une virgule, fait office d'un premier téton, tandis qu'un léger renflement dans la courbe délimitant le torse signale le deuxième. LaPalme prend soin d'inscrire deux petites courbes pour dénoter les plis de l'abdomen. Deux pansements se croisent sur la fesse droite, référence possible aux piqûres que doit recevoir chaque jour un homme qui, comme Maurice Duplessis, est atteint de diabète. Enfin, sa grande tête émerge de son tronc, pyramide renversée structurée par des traits sinueux et des striures courbées qui rappellent la gravure sur bois des expressionnistes. Divisant l'image sur l'axe central, un long nez creuse son chemin tordu devant la bouche et le menton, depuis le pont entre les yeux grands ouverts, mais non voyants.

\footnotetext{
3 Voir Josée Desforges, "La caricature dans Le Devoir entre 1950 et 2010 ", dans Jean Charron, Jean de Bonville et Judith Dubois (dir.), Points de vue sur un journal en mouvement : six études sur Le Devoir (1910-2010), Études de communication publique, $\mathrm{n}^{\circ} 19$, Département d'information et de communication, Québec, Université Laval, 2012, p. 43-64, [En ligne], [www.com.ulaval.ca/fileadmin/ contenu/docs_pdf/articles/etudes_com_publ/ECP19-120418.pdf] (25 mai 2012).
} 


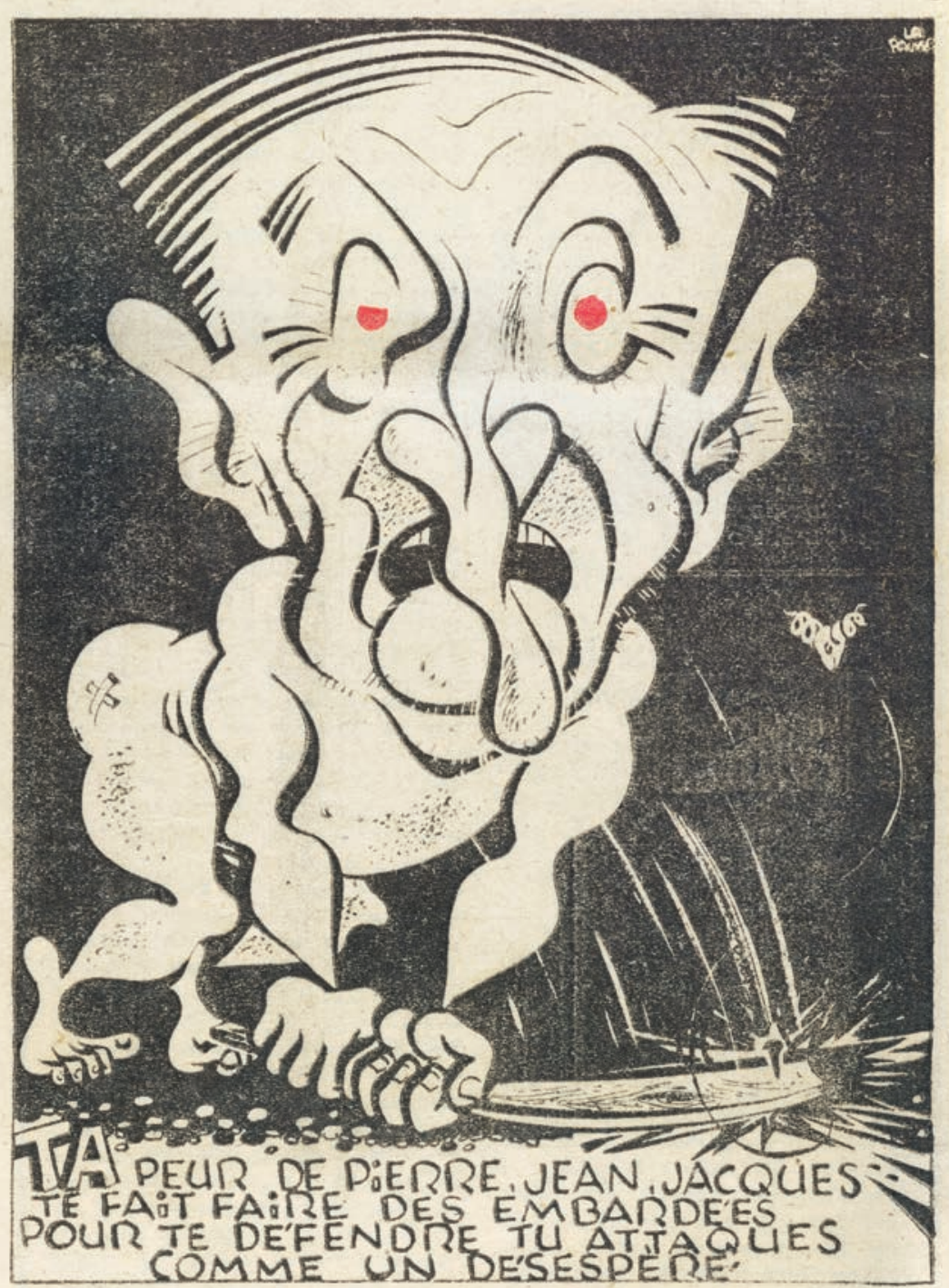

\section{Un valentin au grand Maurice}

Figure 1. «Un valentin au grand Maurice », Vrai, 22 février 1958, p. 1. Avec l'aimable autorisation de la Fondation Robert LaPalme, Montréal. 
Cette caricature de Maurice Duplessis est emblématique de la carrière de Robert LaPalme, le dessin reprenant les trouvailles de ses premiers temps, alors que ses portraits politiques et culturels, construits à partir de formes géométriques très réduites et virtuoses, ornaient les pages de L'Ordre, publié en 1934-1935 par Olivar Asselin (18741937). Mais le vocabulaire est maintenant détourné, intégré à l'attaque coriace, à la fois plus sensuelle mais aussi plus intransigeante qu'a développée LaPalme durant une quinzaine d'années, et qu'il pousse ici dans le sens d'une certaine maladresse, comme pour souligner la férocité et l'immédiateté de la pensée. C'est une image qui s’acharne contre l'emprise qu'exerce Duplessis sur le pouvoir au Québec depuis sa première réélection en 1944. Le Duplessis présenté ici est dénudé, âgé, inconscient, primitif, brutal. Il frappe le sol d'une planche de bois percée d'un clou : l'emport du geste est signalé par des lignes blanches qui traversent la noirceur, creusées comme par une lame dans l'aplat d'encre, de manière à évoquer la trace que laisserait le clou. Au bas du dessin, dans la graphie quasi typographique de LaPalme, aux majuscules évoquant les polices du style Art déco, la légende nous présente cette courte strophe : "Ta peur de Pierre, Jean, Jacques / Te fait faire des embardées / Pour te défendre tu attaques / Comme un désespéré. »

Est-il possible de lire dans ce dernier mot, "désespéré », une évocation de l'auteur français Léon Bloy (1846-1917), par référence au titre de son roman Le désespéré $(1887)^{4}$ ? De ce roman inclassable, qui présente le récit d'un anti-héros, Caïn Marchenoir, double de l'auteur, en ouvrant sur l'aveu d'un parricide par lequel toute la médiocrité de la France bien-pensante, bourgeoise, démocratique, est châtiée sur un ton qui oscille entre la satire et l'apocalypse, seul le vocable du titre fait, en premier lieu, office de coïncidence. Sur ce

\footnotetext{
${ }^{4}$ Voir les textes réunis par Pierre Glaudes, dans les volumes 7 et 8 des cahiers Léon Bloy sur "Le désespéré" ", Caen, Lettres modernes Minard, 2008, coll. " La Revue des lettres modernes ". Nous poursuivons ici une question que nous avons posée d'abord dans nos recherches doctorales (Hardy, A Metropolitan Line, p. 341-351).
} 
seul mot, structure bien mince, repose l'essai qu'on va lire ici. Le désespoir est signe d'un grand péché, du refus du salut. On pourrait se demander déjà si la projection sur la figure de Maurice Duplessis de l'idée du désespoir n'est pas une façon de libérer l'artiste et ses lecteurs d'une condition pénible. En effet, en 1958, l'emprise de Maurice Duplessis semble s'éterniser et gagner une dimension mythique, alors que le Québec arrive au terme de deux longues périodes d'apparente stabilité politique (la génération au pouvoir sous Duplessis suivant la trentaine d'années de dominance libérale des gouvernements de Lomer Gouin et de Louis-Alexandre Taschereau). La résonance de toute évocation ou écho relevant du désespoir, voulue ou non, dépend dans le cas présent, disons-le d'emblée, d'une intuition nourrie avant tout par une réflexion que nous avons menée depuis plus d'une décennie sur le milieu intellectuel et polémique dans lequel a évolué la carrière de Robert LaPalme. Le nom et l'exemple de Bloy hantent ce milieu; c'est une hantise qui se devine notamment dans l'impact qu'a eu Olivar Asselin sur l'ensemble du journalisme de l'époque. Catholique dévot qui épouse pourtant les valeurs du sécularisme dans l'éducation et l'économie nationales, Asselin est un grand défenseur de l'esprit français. Ses armes sont les richesses de la langue et surtout de l'ironie, et parmi ses modèles se dénombrent les hérauts de la presse et des lettres catholiques françaises de la seconde moitié du XIX ${ }^{\mathrm{e}}$ siècle, dont les Louis Veuillot et Ernest Hello, mais surtout Léon Bloy, journaliste, pamphlétaire et " démolisseur de réputations ", romancier, agoniste chrétien farouchement en colère à la fois contre l'Église et contre le régime politique de la Troisième République. Asselin, qui a rendu visite à Léon Bloy peu avant la mort de ce dernier en 1917, cite librement et régulièrement son héros dans ses textes et en discute avec son ami, le romancier et pamphlétaire (presque aussi farouche) Claude-Henri Grignon (1894-1976). Nous nous attarderons ici à l'amplification et à l'exégèse que leurs échanges conferrent au message bloyen. 
Force, virulence, violence : ces mots sont loin de suffire pour caractériser le tempérament littéraire, profondément polémique, de Léon Bloy ${ }^{5}$. Catholique si fervent qu'il en vient à pourfendre l'Église gallicane de son époque, être mystique et ascétique, Bloy est partout dans la vie littéraire de son temps. Collaborateur aux revues satiriques Le chat noir et Gil Blas, auteur de courts textes d'une comédie jaune (Histoires désobligeantes), de romans (Le désespéré, La femme pauvre), d'essais (Propos d'un entrepreneur de démolitions, Le Salut par les Juifs) et de mémoires (Le pèlerin de l'Absolu), Bloy a marqué les combats idéologiques d'une Troisième République qu'il abjurait au même titre que ses institutions sociales, politiques et religieuses. Comme nous le rappelle Pierre Glaudes, l'écriture est pour Bloy une façon de " toujours inventer des figures inouies pour faire entrer "l'intranscriptible" dans l'ordre du langage ".. Au Québec, où l'une des nombreuses filiations à Bloy passe par l'influence du personnalisme de Raïssa et Jacques Maritain, dont Bloy est le parrain, il y a réception constamment renouvelée de cet « intranscriptible ». Dans les Pages de Léon Bloy recueillies par les Maritain et publiées en 1951, les extraits tirés du Désespéré donnent l'ampleur de la crise qui afflige, du sublime vers le ridicule, la personne chrétienne dans la société moderne :

Marchenoir était né désespéré7.

D'autres ont besoin des déconfitures ou des crimes de leur propre vie pour en sentir la nausée. Marchenoir, mieux doué, n’avait

\footnotetext{
${ }^{5}$ Les études bloyennes connaissent un nouvel essor à partir des années 1990. On consultera la série Cahiers Léon Bloy éditée à la Revue des lettres modernes par la Société des études bloyennes entre 1988 et 1994 . Les historiens de la littérature Pierre Glaudes et David Cocksey ont créé un site Web où ils proposent un survol historiographique détaillé des bibliographies et études bloyennes : Léon Bloy, [En ligne], [http://barbey.chez.com/bloy/] (25 mai 2012). Voir aussi Pierre Glaudes (dir.), Léon Bloy au tournant du siècle, Toulouse, Presses universitaires du Mirail, 1992.

6 Pierre Glaudes, "Biographie ", sur le site Léon Bloy.

7 Raïssa Maritain (dir.), Pages de Léon Bloy, présentation de Jacques Maritain, Paris, Mercure de France, 1951, p. 77.
} 
eu de peine que de venir au monde... ${ }^{8} \mathrm{Il}$ avait fait sa première communion sans malice et sans amour. Les deux seules facultés qui parussent vivantes en lui, [...], la mémoire et l'imagination, avaient tout simplement reçu cette vague empreinte littérale du symbolisme chrétien que de sacrilèges entrepreneurs jugent suffisante pour être admis au bachot de l'Eucharistie. Aucun débitant de formules ne s'étant avisé de s'enquérir de son cœur, le pauvre enfant n'avait pu rien garder de ce pain mal cuit et, comme tant d'autres, l'avait revomi presque aussitôt sur ce chemin verdoyant de la quinzième année où l'on voit rôder le grand lion à tête de porc de la Puberté. Marchenoir avait la réprobation scatologique... il voyait le monde moderne, avec toutes ses institutions et toutes ses idées, dans un océan de boue. C'était, à ses yeux, une Atlantide submergée dans un dépotoir. Impossible d'arriver à une autre conception... il se trouvait, habituellement, dans la nécessité la plus inévitable de se détourner de la vie contemporaine, ou de l'exprimer en répulsives images, que l'incandescence du sentiment pouvait, seule, faire applaudir. L'article qu'il avait [écrit] sur le scandale de la publicité pornographique était, en ce genre, un tour de force inouï. C'était un Vésuve d'immondices embrasés $[s i c]^{10}$.

On n'a pas nécessairement à s'en tenir à l'influence des Maritain ni à l'association admirative qu'entretiennent Asselin et Grignon pour prendre la mesure de la présence de Bloy au Québec. En 2010, Éric Walbecq dresse un portrait exhaustif de la réception de Bloy dans les milieux religieux, intellectuel, littéraire et même politique - pour peu qu'on puisse dissocier ces quatre milieux - depuis 1911 jusqu'à la fin des années 1970, notamment dans le cadre des associations d'amis qui ont développé, après la Seconde Guerre mondiale, une branche importante des études bloyennes internationales ${ }^{11}$. Cependant, cette récente étude ne s'est pas attardée au contact que

${ }^{8}$ Ibid.

${ }^{9}$ Ibid., p. 81.

${ }^{10}$ Ibid., p. 105.

${ }^{11}$ Éric Walbecq, "Présence de Léon Bloy au Canada », dans Marie-Pier Luneau et al. (dir.), Passeurs d'histoire(s) : figures des relations France-Québec en histoire du livre, Québec, Les Presses de l'Université Laval, 2010, p. 345-353. 
nous cherchons à évoquer ici. C'est pourquoi nous proposons de revisiter ce moment intense de transfert dans les années 1930, au moment où la figure de Bloy devient signifiante pour l'imaginaire canadien-français par la concertation amicale entre deux acteurs principaux, Asselin et Grignon. Ensemble, les deux amis semblent en appeler de leurs vœux les plus fervents qu'une violence bloyenne puisse secouer le Canada français - dans sa culture littéraire et aussi dans son comportement social. L'attention que nous voulons porter à l'examen de ce moment peut sembler disproportionnée, car après tout, nous cherchons à comprendre comment s'est dessinée une caricature plus violente que d'habitude en 1958. Les raisons sont sans doute du côté des mutations qui se sont opérées dans la société depuis les années 1930, à travers une guerre mondiale et ses horreurs, à travers la guerre froide et la menace de la destruction atomique, à travers toute une série de facteurs qui expliqueraient le recours à la représentation de la nudité grotesque d'un homme politique. Lobjectif ici est de ne pas perdre de vue la force latente qu'a pu avoir un appel lointain - révolu, peut-être jamais satisfait, si souterrain soit-il - à la violence.

Comme nous plaçons Robert LaPalme au début et à la fin de l'histoire que nous avons à narrer ici, il convient de revenir sur la trentaine d'années durant lesquelles il a contribué aux différents journaux qui l'ont accueilli. L'analyse des relations nouées entre le caricaturiste et ses éditeurs correspond assez bien au modèle classique d'études sur la caricature et le rôle de cette forme satirique dans la sphère publique, depuis le XVIII ${ }^{\mathrm{e}}$ siècle en Europe et dans les Amériques ${ }^{12}$. Selon ce parti pris méthodologique, les journaux et leurs caricaturistes sont étudiés selon l'identité que leur confèrent les

${ }_{12}$ Voir David Kerr, Caricature and French Political Culture, 1830-1848: Charles Philipon and the Illustrated Press, Oxford, Clarendon Press, 2000; Mark Hallett, The Spectacle of Difference: Graphic Satire in the Age of Hogarth, New Haven/Londres, Yale University Press, 1999; Diana Donald, The Age of Caricature: Satirical Prints in the Reign of George III, New Haven/Londres, Yale University Press, 1996; Ségolène Le Men (dir.), L'art de la caricature, Paris, Presses universitaires de Paris-Ouest, 2011. 
éditeurs. Dans le cas de Robert LaPalme, dont la carrière correspond à l'époque circonscrite par les gouvernements de Maurice Duplessis (au pouvoir d'abord de 1936 à 1939, et ensuite pour quatre mandats successifs entre 1944 et sa mort en 1959), il s'agit des journaux d'Olivar Asselin (Le Canada, L'Ordre et La Renaissance, 1933-1935), de Jean-Louis Gagnon dans sa période de droite (Le Journal et L'Événement-Journal, 1937-1939), d'Eugène L'Heureux (L'Action catholique, 1940-1943), d'Edmond Turcotte (Le Canada, 1943-1951), de Gérard Filion et d'André Laurendeau (Le Devoir, 1952-1959), auxquels il faut ajouter la participation de LaPalme aux journaux de Jean-Charles Harvey (Le Jour, où il signe des dessins en 1945), de Jacques Hébert (Vrai, 1954-1959) et d'un Jean-Louis Gagnon devenu libéral à $L a$ Presse et au Nouveau Journal (1959-1962). Le recensement attentif des dessins du caricaturiste dans leur contexte permet de comprendre quelle place et quelle autorisation on accordait à la satire visuelle dans la constitution des discours des journaux. Or on veut saisir comment cette autorisation pouvait ensuite contribuer à l'instauration d'un climat duquel les dessins et légendes de LaPalme tiraient leur sens ${ }^{13}$.

Partant d'une analyse iconographique classique de la caricature intitulée Un valentin au grand Maurice, réalisée par Robert LaPalme à la fin de sa carrière, le présent article se propose d'examiner comment cette production serait l'expression d'un phénomène de transfert culturel beaucoup plus difficile à saisir, phénomène par lequel certains aspects de l'écriture de Léon Bloy sont transposés dans le discours culturel (aussi bien littéraire que graphique) québécois et dans lequel Olivar Asselin et Claude-Henri Grignon, séparément et conjointement, ont joué le rôle essentiel de passeurs. Dans ce cas-ci, ce sont à la fois le climat polémique de la Troisième République française et son influence sur les discours politiques et, plus largement, sociaux et religieux au Québec après 1900 qui sont largement en cause. Les polémiques, pamphlets et caricatures impitoyables de la France

13 Cette étude se trouvait notamment au centre de nos recherches doctorales (Hardy, A Metropolitan Line, chapitres 2 et 5). 
anticléricale, anti- et pro-dreyfusarde, des Grelot, Assiette au Beurre, Psst! et de nombreuses autres revues satiriques, sont bien accessibles aux générations canadiennes-françaises qui grandissent en parallèle, des années 1870 aux années 1930. Mais, exception faite des journaux fascistes québécois des années 1930 (principalement Le Goglu, 19291934 et La Nation, 1936-1939), la violence qui caractérise ces polémiques françaises ne trouve que peu d'échos au Québec. L'hypothèse retenue ici, somme toute assez simple, est que l'environnement intellectuel - et spirituel - façonné par Asselin et Grignon, lecteurs qui affectionnent la presse polémique française de leur jeunesse et, surtout, l'exemple de la littérature radicale dont Léon Bloy est l'un des plus fervents adhérents, ne résonnera qu'une fois que Robert LaPalme se permettra d'adopter la même position. Les conditions pour le faire ne se mettent en place que vers la fin de la vie de Maurice Duplessis qui maintient, malgré sa déchéance corporelle et politique, et malgré l'incrédulité de ses antagonistes - notamment LaPalme, les rédacteurs du Devoir André Laurendeau et Gérard Filion et le chef libéral Georges-Émile Lapalme -, son emprise sur le pouvoir et sur l'imaginaire du Québec. C’est devant cette apparente invincibilité que les propos les plus acharnés de polémistes comme LaPalme se déchaîneront enfin contre l'ordre mythique du Québec.

En bon historien de l'art, nous partirions normalement à la recherche d'un programme, d'une espèce de contrat par lequel cette violence latente, enfin déchaînée, s'expliquerait, se justifierait. Faute d'une trace concrète - d'une note de la main de Jacques Hébert, d'une réflexion confiée à quelque journal intime par Robert LaPalme dont les archives personnelles restent à dépouiller -, c'est par la reconstruction du climat intellectuel que nous avançons. Et en fait, nous devons surtout nous livrer à la recherche des traces d'une influence qui est restée largement tacite. Nous acceptons d'y aller d'une méthode qui dépend beaucoup plus de relevés faits à partir de circonstances et de coïncidences. Les pistes pouvant mener aux traces qui nous intéressent - traces qui, elles-mêmes, " prennent " souvent des allures fugitives, il faut le dire - seront d'abord les activités d'Olivar 
Asselin et de Claude-Henri Grignon, lecteurs et interprètes actifs de Bloy, qui s'unissent ensuite par leur appréciation commune de celui qui s'est nommé « le Mendiant ingrat », ou « le Pèlerin de l'Absolu ». Ainsi, "suivre les traces ", c'est d'abord les suivre chez Asselin et ensuite chez Asselin et Grignon ensemble, pour terminer avec Grignon qui défend la mémoire et de Bloy et de son ami Asselin. Enfin, comme Asselin et Grignon n'ont été que deux parmi les plus remarquables exégètes de Bloy au Québec dans la période qui correspond à celle du régime de Duplessis, nous poursuivrons l'enquête sur la fortune de Bloy au cours de cette période, ne serait-ce que pour rendre explicite, et pour le sortir de l'ombre, un climat qui pourrait nous permettre de revenir à notre dessin. La caricature politique pose des défis à l'histoire de l'art, surtout parce qu'elle s'insère dans une temporalité très particulière, celle qu'évoque de manière si poignante Georges-Émile Lapalme (1907-1985, chef du Parti libéral du Québec, 1950-1958), dans les Mémoires qu'il publiera au tournant des années 1970. Or un ancien chef du Parti libéral est rarement admis comme autorité dans la méthode des historiens de l'art au Québec... mais c'est aussi vers les balises littéraires de la culture politique de notre pays que nous devons nous orienter pour rendre compte des forces qui animent et traversent l'image. La méthode est non moins vraie pour la caricature qu'elle ne l'est pour la peinture automatiste. Dans les pages qui suivent, notre méthode consistera simplement, disonsle encore, à organiser les traces que nous avons décelées pour tenter de saisir ce qu'elles montrent.

\section{Passeur de Bloy : Olivar Asselin, l'éditeur de caricatures}

Journaliste de combat très actif dans le mouvement de jeunes nationalistes qui suivent Henri Bourassa dès l'automne 1899, Olivar Asselin a été dans sa jeunesse franco-américaine un lecteur assidu de la presse polémique et catholique française de la Troisième République $^{14}$. Il y trouve, à la mesure de ses propres convictions à

${ }^{14}$ Hélène Pelletier-Baillargeon, Olivar Asselin et son temps, t. 1 : Le militant, SaintLaurent, Fides, 1996, p. 70-90. 
l'égard d'une presse libre qui cherche à défendre l'intégrité de la langue et de la civilisation françaises, l'expression d'une ardeur qu'il souhaiterait faire vivre au Canada français. Il se fera, en tant que journaliste, maître du ton ironique, caustique et satirique que véhicule cette presse française, ton qu'il mettra au service de la défense des droits de la collectivité canadienne-française et d'une société inspirée par la foi catholique, mais organisée autour d'institutions séculaires.

Le plus vociférant parmi les auteurs français à marquer l'imaginaire d'Olivar Asselin est Léon Bloy. Asselin découvre Bloy, Barbey d'Aurevilly et Ernest Hello, auteurs d'une droite catholique farouche, à peu près en même temps. Il partage avec ces derniers une préoccupation fondamentale pour le détournement que subit la foi sous le poids des structures d'une société capitaliste et se livre comme eux à la critique du rôle de l'Église catholique dans la société15. Fort d'une position éthique prononcée, Asselin privilégie dans ses écrits le recours au mode satirique et s'assure de la présence de plusieurs indicateurs de ce mode dans ses journaux. L'ironie et la satire sont au cœur de sa conception de la langue française et de l'ordre social, éléments primordiaux du nationalisme rationnel et humaniste qu' il va prôner dans son journalisme. Ce nationalisme est aussi défini par une critique constante, précise et acerbe des liens entre le système politique fédéral du Canada et l'impérialisme britannique, liens qui se concrétisent à partir de l'aventure coloniale de la guerre des Boers. On voit l'importance qu'il accorde à la démonstration d'une démarche satirique qui soit textuelle et visuelle, dès les premiers numéros du Nationaliste qu'il fonde en 1904, par les caricatures qui ornent la une du journal. Le Nationaliste reprend une habitude adoptée par le journal Les Débats, fondé en 1899 au lendemain de l'adoption par le gouvernement de Wilfrid Laurier d'une politique de participation volontaire à la guerre des Boers, décision qui mène à la création du nouveau mouvement nationaliste. Ce faisant, Les Débats et Le

${ }^{15}$ Ibid., p. 617. 
Nationaliste transposent dans la presse d'information et d'opinion ce qui était coutumier dans la presse satirique depuis longtemps. De la même manière, la caricature est adoptée de façon permanente (mais rarement à la une) par les grands journaux tels que The Montreal Daily Star (dès 1899), La Patrie (dès 1903) et La Presse (dès 1905). Par ailleurs, Asselin verra cette pratique adoptée à son tour par son ami Jules Fournier (1884-1918) quand celui-ci fonde L'Action en 1911 (il en sera de même pour Le Pays, fondé par Godfroy Langlois en 1910). Étonnamment, Le Devoir, qui voit le jour en même temps et auquel ont brièvement collaboré Asselin et Fournier dans les premiers mois du journal, résistera à la caricature jusqu'à l'arrivée de Robert LaPalme en 1952. Dans sa biographie d'Olivar Asselin, Hélène Pelletier-Baillargeon nous donne une idée du climat créé au Canada français par les amis Asselin et Fournier, à l'image de ce que Bloy fait en France :

Les deux pamphlétaires, Asselin surtout, se sont retrouvés dans les accents imprécateurs et le style somptueux d'un Léon Bloy. D'abord, comme eux, journaliste de combat au Gil Blas et au Chat noir, l'écrivain périgourdin aux métaphores de visionnaire connaît, en France, une existence de misère et d'isolement indescriptible. Cette situation semble délibérément entretenue par une sorte de volonté farouche de la part du pamphlétaire de s'en prendre à toutes les écoles de pensée et à la plupart des institutions en place ${ }^{16}$.

Olivar Asselin continue à recourir aux modes de l'ironie et de la satire alors même qu'il s'éloigne quelque peu du journalisme. En 1910, il signe la préface du recueil de poèmes "Mignonne, allons voir si la rose "... est sans épines ${ }^{17}$, parodie de la littérature du terroir écrite par Guy Delahaye (pseudonyme du médecin Guillaume Lahaise, 18881969), poète et futur collaborateur de la revue Le Nigog, où, en 1918, plusieurs des premiers jalons de la modernité culturelle du Québec

${ }^{16}$ Ibid., p. 616.

17 Guy Delahaye (pseudonyme de Guillaume Lahaise), "Mignonne, allons voir si la rose "... est sans épines, préface d'Olivar Asselin, Montréal, C. Déom, 1912. 
seront posés ${ }^{18}$. Dans sa préface, Asselin demande : «si n'était l'amitié personnelle qui nous lie, [Delahaye] ne serait[-il] pas tenté de me compter parmi les cochons que, sans comme Léon Bloy les nommer par leur nom, il invite aujourd'hui à aller voir avec Mignonne "si la rose est sans épines" 1 . " L'invitation qu'interprète ici Asselin conduirait la littérature canadienne-française, telle que lue quelques années plus tard par $\mathrm{M}^{\mathrm{gr}}$ Camille Roy, vers une situation bien néfaste. Dans son Manuel d'histoire de la littérature canadienne-française de 1918, édition publiée la même année que Le Nigog, c'est plutôt la poésie du terroir de Blanche Lamontagne qu'encense Roy, poésie qu'il trouve exemplaire d'une saine littérature, fondée sur de saines idées, marquée par une démarche laborieuse et donc pleine de mérite. Roy critiqua les excès du symbolisme en littérature, associant l'imprécis, l'indéfini que favorise ce mouvement à un état d'âme insalubre et même impie ${ }^{20}$. Le fait que Le Nigog soit loin de faire l'unanimité de la critique se confirme quelques années plus tard, dans un tout autre registre, par Claude-Henri Grignon. Grignon accuse Le Nigog d'être Le Nigaud, peuplé par des décadents, des symbolistes qui «s'acharnent $[\ldots]$ à changer le sens et le rapport des $\operatorname{mots}^{21} »$. Un par un, Grignon en repère les responsables, comme ce Guy Delahaye

${ }^{18}$ Voir l'analyse du Nigog faite par Esther Trépanier dans le chapitre intitulé "Le Nigog : une première expression de la modernité " dans son ouvrage Peinture et modernité au Québec 1919-1939, Québec, Nota bene, 1998, coll. "Essais critiques ", p. 34-40.

19 Delahaye, "Mignonne, allons voir si la rose "... est sans épines, $\mathrm{p}$. xiii. Il peut être utile de rappeler la dédicace de l'auteur : «À ceux / d'un dédain absolu pour la médiocrité, / d'un rire immense devant la bêtise " (p. xi).

${ }^{20}$ Ce résumé du projet de Roy est fondé sur la lecture de Marie-Andrée Beaudet, Langue et littérature au Québec 1895-1914, Montréal, L'Hexagone, 1992, notamment les chapitres "Champ littéraire et champ linguistique " (p. 37-58) et "Camille Roy : le grand programmateur» (p. 87-106). L'histoire des éditions du Manuel est discutée dans Lucie Robert, Le manuel d'histoire de la littérature canadienne-française de $M^{g r}$ Camille Roy, Québec, Institut québécois de recherche sur la culture, 1982.

21 Robert Lahaise, Une histoire du Québec par sa littérature, 1914-1939, Montréal, Guérin, 1998, p. 350 et n. 209. 
qui « va s'épuisant dans l'énormité de son œuvre insignifiante ${ }^{22}$ ". Même si Grignon devient par la suite l'un des amis les plus dévoués d'Asselin, il montre ici, sur un ton bloyen, une opposition à toute possibilité de remise en question, ne serait-ce qu'à des fins esthétiques, de la stabilité du langage, peut-être parce qu'il s'agit précisément non seulement de langage, mais de la langue française comme telle. Or Asselin défend la possibilité d'un jeu opéré sur le langage, comme celui qui habite les poèmes de Delahaye. C'est une position que la richesse de la langue bloyenne, déstabilisante, pouvait peut-être encourager.

Il y aurait certainement lieu d'examiner de plus près l'impact de la Première Guerre mondiale sur les discours culturels au Canada et, plus particulièrement, de voir comment la démarche satirique constitue une stratégie de plus en plus normalisée pour une société en proie aux déchirements identitaires dont témoignent, après la guerre des Boers (1899) et la question des écoles françaises de l'Ontario (1912), les épreuves de la conscription (1917). Asselin, qui va à contre-courant en s'enrôlant dans l'armée canadienne avec le souhait de former un bataillon francophone, profite de son séjour en France pour rendre visite à son héros, Léon Bloy, quelques mois avant la mort du Pèlerin de l'Absolu. Le rendez-vous symbolique suggère la transmission qu'Olivar Asselin assure déjà, et assurera encore, de l'exemple de Bloy.

Tout comme la querelle qui oppose les " exotiques " aux écrivains du terroir, on constate comment la verve satirique se rattache elle aussi à différentes positions dans les polémiques sur la culture, et qu'elle prend parti pour ou contre l'autonomie des éléments de l'œuvre artistique. Dans le même temps, le climat culturel montréalais de l'époque ne semble pas (du moins jusqu'ici) avoir laissé la trace d'une volonté comparable en arts visuels, même dans la caricature des journaux quotidiens, exception faite de La Bombe, publiée durant l'année 1909 par des étudiants du milieu de la musique ${ }^{23}$.

22 Ibid.

${ }^{23}$ Voir La Bombe : journal satirique paru en 1909 à Montréal, sous la direction de Sandria P. Bouliane et Jasmin Miville-Allard, Montréal, Moult Éditions, 2011 [1909], coll. « Inauditous ». 
C'est avec Robert LaPalme qu'Asselin trouvera, en 1933, un dessinateur à la hauteur de sa vision. Après la Première Guerre mondiale, la caricature étant bien établie dans la presse quotidienne, la pratique s'est standardisée autour des figures d'Albéric Bourgeois (1876-1962) et d'Arthur George Racey (1870-1941). Un signe de renouveau paraît en 1933 dans l'Almanach de la langue française, alors que l'éditeur Albert Lévesque y publie des "silhouettes " de " petits classiques canadiens". Ce sont là parmi les premiers dessins publiés de la main du jeune caricaturiste Robert LaPalme ${ }^{24}$. LaPalme y présente une caricature renouvelée, stylistiquement novatrice au moment où le culte de la célébrité et de la personnalité connaît un net essor dans le journalisme canadien-français. Dans une notice critique sur l'Almanach qu'il fait paraître dans Le Canada ${ }^{25}$ dont il est alors le rédacteur en chef, Olivar Asselin évoque le plaisir qu'il éprouve à voir ainsi caricaturé son compère journaliste Louis Francoeur (1895-1941), rédacteur en chef, de 1929 à 1936, du Journal de Québec et coauteur, avec Philippe Panneton (1895-1960), d'une série de parodies, Littératures à la manière de..., parues en $1924^{26}$. Asselin compare Francoeur à ses héros : Ernest Hello et Léon Bloy. Asselin est surtout content de lier ainsi trois auteurs bien différents qui auraient pour but commun de déstabiliser le consensus trop pragmatique de la politique, que ce soit en France ou au Québec. Asselin donne aussi une idée des limites éprouvées par tout caricaturiste :

À signaler aussi une galerie de portraits esquissés, ou plutôt indiqués, par La Palme [sic], jeune artiste de talent qui ne fait

${ }^{24}$ Hardy, A Metropolitan Line, p. 65-90.

25 Olivar Asselin, "L'almanach de la langue française ", Le Canada, 30 décembre 1933, p. 12.

26 Louis Francoeur et Philippe Panneton, Littératures... à la manière de... Henri Bourassa - René Chopin - Valdombre - Henri Letondal - Paul Morin - l'abbé Camille Roy - La Presse - l'abbé Groulx-Madeleine - Victor Morin-Marcel Dugas - Blanche Lamontagne - Gustave Comte - Édouard Montpetit - l'abbé Blanchard, Montréal, Édouard Garand, 1924, coll. "Éditions des essais ». 
« ressemblant » qu’à condition de « défigurer » : [...] parmi ces défigurés, beaucoup de "gloires nationales». Il en est cependant (ne les nommons pas : l'époque n'est pas aux personnalités... désobligeantes) dont la tête, défigurée ou non, ressemblera toujours à un vase... égyptien ${ }^{27}$.

En rattachant la " personnalité " à la " désobligeance ", Asselin fait surtout référence à Bloy, dont les Histoires désobligeantes ont été publiées de 1892 à 1894 dans l'hebdomadaire parisien Gil Blas ${ }^{28}$. Bloy poursuivait alors une relation souvent tumultueuse avec le milieu de l'humour et de la satire qui avait notamment marqué son entrée au Chat noir, journal éponyme du célèbre cabaret montmartrois de notoire souvenir des années $1880^{29}$. Comme le signalera Robert LaPalme dans le cadre d'un souvenir publié en 1978, c'est surtout l'atmosphère du Chat noir qu'avait voulu faire revivre le milieu des étudiants et des boîtes de nuit du Quartier latin à Montréal à la fin des années $1920^{30}$. Mais pour Olivar Asselin, l'heure est surtout à la recréation de l'esprit français dans le Québec des années 1930. Il quitte Le Canada en janvier 1934 pour lancer L'Ordre le 6 avril suivant. Le sous-titre est clair : "Quotidien de culture française et de renaissance nationale ». Son premier dessinateur, Essel $^{31}$, est présent dès le premier numéro, avec des dessins reproduits au pied de la première page, habituellement dans la colonne centrale.

27 Asselin, "L'almanach de la langue française », p. 12.

28 Pierre Glaudes, "Histoires désobligeantes, histoires drôles? ", dans Joseph Royer et Bernard Sarrazin (dir.), Léon Bloy, t. 2 : Le rire de Léon Bloy, Paris, Lettres modernes Minard, 1994, coll. "Revue des lettres modernes. Série Léon Bloy », p. 77-109.

29 Pour un précis du cabaret Le Chat noir et de son fondateur, Rodolphe Salis, on se référera à Mariel Oberthür, Le Chat noir 1881-1897, Paris, Musée d'Orsay, 1992, coll. "Les dossiers du Musée d'Orsay ". La relation entre Léon Bloy et Le Chat noir a été examinée par François Caradec ("Deux "fumistes” : Léon Bloy et Alphonse Allais ") et Michèle Fontana ("Léon Bloy et Le Chat noir ", dans Royer et Sarrazin (dir.), Léon Bloy, p. 25-34 et 35-48).

30 Robert LaPalme, dans Léon-A. Robidoux, Albéric Bourgeois, caricaturiste, Montréal, VLB éditeur et Médiabec, 1978, p. 18.

${ }^{31}$ Ce nom semble être un pseudonyme façonné autour de deux initiales, S. L.; l'identité de l'artiste demeure pour le moment inconnue. 
Dès le mois de mai, la caricature remonte la colonne centrale pour s'abriter sous le titre, avant de redescendre au pied de la page le 17 juillet. Le 26 juillet, Robert LaPalme fait sa première apparition avec une image qui détonne par rapport aux dessins très soignés et prenants d'Essel, dont la structure privilégiait jusque-là la mise en scène de commentaires satiriques dans des environnements conçus pour renvoyer à des réalités connues des lecteurs. LaPalme y va, non d'une mise en situation, mais d'un portrait qui remet en question le vocabulaire même de la représentation visuelle et de la ressemblance dont dépend la caricature. En cela, il s'inscrit dans la lignée des explorations visuelles que mènent les Miguel Covarrubias (19041957), Paolo Garretto (1903-1989) et Al Hirschfeld (1903-2003) dans les périodiques new-yorkais de l'époque (New Yorker, Vanity Fair, New York Times). Dans la première des caricatures qu'il livre à L'Ordre le 26 juillet 1934, intitulée M. Ramsay MacDonald (alors chef du Parti travailliste de la Grande-Bretagne), LaPalme semble travailler la poignée d'une canne, taillée en silhouette. Ses dessins sont publiés en alternance avec des caricatures empruntées aux journaux français (notamment celles du dessinateur Jean Sennep, 1894-1982) et américains.

Parmi les choix les plus remarquables faits par Asselin, se trouve celui de reprendre, pour un texte intitulé «Le Christ au pays des gaz » (28 juillet 1934), la célèbre caricature de l'artiste allemand Georg Grosz (1893-1959), Ferme-la et continue à servir, qui fait partie d'une série de plusieurs dessins critiques présentés comme décor de la pièce Le Bon Soldat Chvéïk de Jaroslav Hašek et repris dans le recueil Hintergrund - en français "Arrière-plan » - que Grosz publie en 1928. Le dessin, au trait informel acharné, qui semble égratigner la feuille du journal, représente un Christ crucifié qui porte un masque à gaz et des bottes de soldat. Ferme-la et continue à servir, ainsi que les autres dessins de Grosz, avaient valu à l'artiste, dès 1928, une poursuite en justice pour blasphème contre les autorités allemandes. Le procès se poursuit jusqu'en 1932. En 1933, quelques jours avant l'avènement au pouvoir d'Adolf Hitler, l'artiste émigre aux États-Unis 
où il connaît une célébrité immédiate, entre autres, grâce à un reportage dans l'hebdomadaire Time qui met en valeur l'épisode du procès qui lui a été intenté. Le texte publié un an plus tard dans L'Ordre, signé " Gallus ", fait état des expériences menées à Paris et à Londres par les autorités nazies. Au pouvoir depuis un an, celles-ci veulent mesurer l'impact des « attaques aériennes par les gaz " qui caractériseront la "prochaine guerre». Et l'auteur de conclure : "Cette œuvre de destruction naît dans l'Allemagne hitlérienne, où la frénésie des foules a cloué le Christ sur une croix gammée. Le dessin de Grosz, que nous reproduisons ci-contre, résume cette terrible injure au christianisme que constitue le néo-paganisme belliqueux des Germains $^{32}$. " L'interprétation de cette caricature, faite par L'Ordre en 1934, relève d'un certain anachronisme, car Grosz ciblait la société allemande d'avant l'avènement des nazis. Cette représentation du Christ, figure hautement iconique et symbolique, dans un milieu catholique peu habitué à de telles déformations modernistes, est pour le moins surprenante. Nous ne pouvons d'ailleurs qu'imaginer comment un catholique de la trempe d'Asselin a pu apprécier l'ironie de cette référence à un Christ qui doit « la fermer » et " continuer à servir ». C'est là un choix qui s'aligne sur la lamentation bloyenne du Sauveur transfiguré par la condition moderne.

C'est dans ce climat que les caricatures de Robert LaPalme commencent à paraître de plus en plus régulièrement à la une du journal à partir du mois d'août, poursuivant dans la veine annoncée avec son M. Ramsay MacDonald du 26 juillet. Remarquablement, à l'audace double du sujet et du style qui a infiltré L'Ordre par le choix du dessin de Grosz, LaPalme y oppose une audace tout autre, fondée, comme on l'a vu, sur la virtuosité stylistique. À partir du 28 septembre, il lance ses " gloires nationales ». Celles-ci portent, comme titre audessus du dessin, le nom d'une discipline ou d'un champ d'expertise et, en légende, en-dessous, le nom du personnage, comme c'est le

32 Gallus (pseud.), "Le Christ au pays des gaz ", L’Ordre, $1^{\text {re }}$ année, n 117 (28 juillet 1934), p. 1. 
cas du portrait de l'abbé Lionel Groulx qui inaugure la série (voir la figure 2). Ainsi, à l'Histoire (Lionel Groulx), succèdent les dessins de la Flore canadienne (le frère Marie-Victorin), et, le 8 novembre, du Journalisme (Olivar Asselin lui-même, voir la figure 3). La participation de LaPalme à la série prend fin le 9 janvier 1935, en raison de son mariage et, surtout, de son départ de Montréal pour New York. Son successeur, René Chicoine, lui rendra d'ailleurs hommage dans deux dessins, dont le premier se veut un exemple de « nos futures gloires nationales ".

La centaine de dessins de Robert LaPalme publiés dans les pages de L'Ordre constituent un moment clé dans l'évolution de sa carrière. Ces caricatures représentent aussi le versant joyeux, rationnel, spirituel (au sens d'amusant, de fin) du climat satirique revendiqué par Asselin et, il faut le dire, qui s'apparente en général à l'idée que se font, dans cette décennie, les auteurs canadiens-français de ce que doivent être la caricature et l'ironie.

Nous avons relevé ailleurs les balbutiements du discours critique suscité par les dessins de LaPalme lorsqu'il les présente dans des expositions au cours des mêmes années ${ }^{33}$. La virtuosité naissante de Robert LaPalme - que Bernard Valiquette et Henri Girard, entre autres, rapprocheront de l'esprit français par les qualités d'innovation, de simplicité et de clarté - donne le ton convenable d'une ironie encore assez légère ${ }^{34}$.

"Défigurant "sans méchanceté apparente, les caricatures de LaPalme semblent dépourvues de tout motif violent, et elles sont certainement étrangères à l'esprit de Ferme-la et continue à servir, dessin " bloyen » dont il a fallu taire le langage en supprimant son titre original, ce qui a ouvert l'image à une interprétation sensiblement différente. Sans doute est-ce ce qu'il faut pour garantir l'adhésion du

33 Dominic Hardy, «Un modernisme de bon aloi : réception critique et politique de la caricature en 1937 ", dans Denis Saint-Jacques et Yvan Lamonde (dir.), 1937 : un tournant culturel, Québec, Les Presses de l'Université Laval, 2009, p. 299-312.

34 On consultera l'examen détaillé de la réception critique des débuts de la carrière de LaPalme dans Hardy, A Metropolitan Line, p. 60-89. 


\section{L'HISTOIRE}

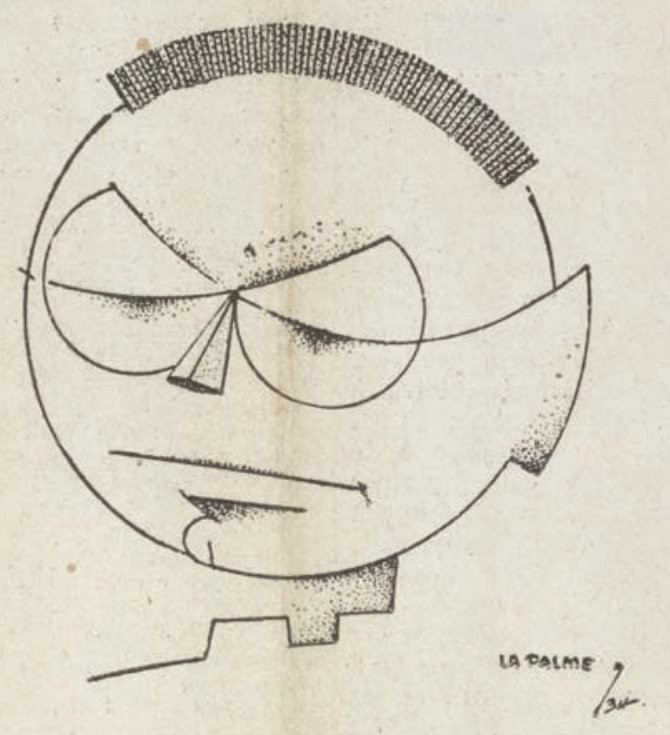

\section{Lionel Groulx}

Figure 2. "L'Histoire / M. Lionel Groulx ", L'Ordre, 28 septembre 1934, p. 1. Avec l'aimable autorisation de la Fondation Robert LaPalme, Montréal. 


\section{LE JOURNALISME}

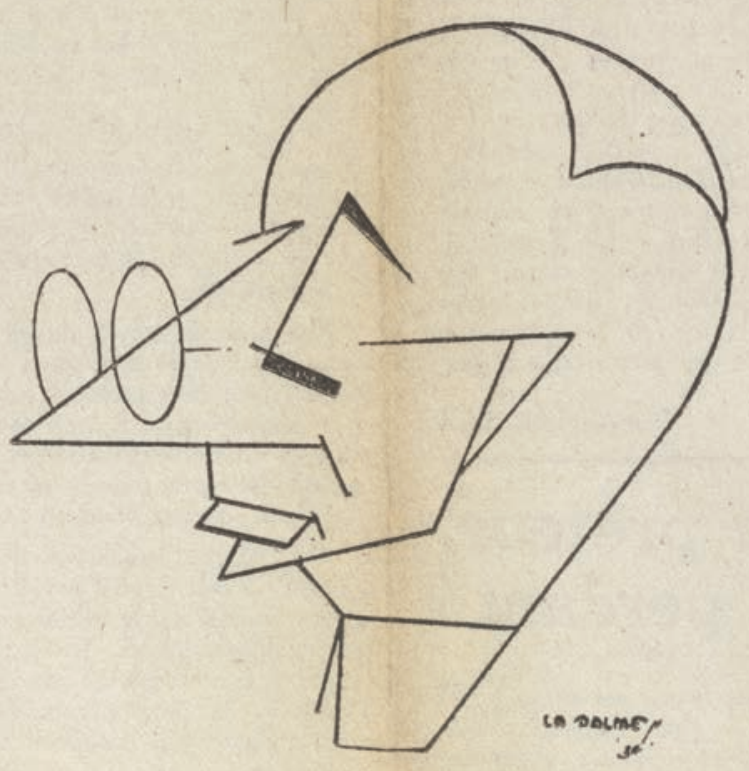

\section{Olivar Asselin}

Figure 3. «Le Journalisme / M. Olivar Asselin », L'Ordre, 8 novembre 1934, p. 1. Avec l'aimable autorisation de la Fondation Robert LaPalme, Montréal. 
public canadien-français à une démarche critique rationnelle. Et c'est ainsi que la caricature de célébrité de LaPalme adapte à l'environnement du Québec un style américain, voire international, mais selon une modulation qui se veut "française ». C'est ce qui inscrit la caricature de LaPalme dans les paramètres de L'Ordre. Et pourtant, si nous considérons ce mot "Ordre " dans le contexte de sa représentation dans l'œuvre de Bloy, on peut penser que l'usage qu'en fait Asselin est, après tout, tout aussi ironique. Le versant plus sombre de la caricature, plus proche de l'esprit acharné et désespéré de Bloy, existe aussi pour Asselin, mais surtout ailleurs, parallèlement à l'aventure de L'Ordre.

\section{Olivar Asselin et Claude-Henri Grignon}

C'est dans sa relation avec Claude-Henri Grignon, auteur du roman Un homme et son péché (1933) et, plus tard, scénariste des Belles Histoires des pays d'en haut diffusées à partir de 1939 à la radio ${ }^{35}$, qu'on retrouve l'expression de ce versant. Avant de prendre la relève d'Asselin, son maître bien aimé, décédé en $1937^{36}$, en consacrant de nombreuses pages de ses Pamphlets de Valdombre à Léon Bloy dans les années 1937-1943, Grignon est surtout cet auteur qui souffre de ne pouvoir mener à bien sa carrière, fragile dans les premiers temps qui suivent la publication d'Un homme et son péché. Grignon était alors résolument associé à Léon Bloy. En 1924, dans l'ouvrage déjà évoqué, Littératures à la manière de..., les auteurs Louis Francoeur et Philippe Panneton (qui devient célèbre sous le nom de Ringuet lors

35 La série est diffusée sur les ondes radio de la CBC de 1939 à 1962, puis à CKVL de 1963 à 1965. Elle passe à la télévision de Radio-Canada de 1956 à 1970. Voir "Parcours thématique de Claude-Henri Grignon : Un homme et son péché... l'œuvre de Claude-Henri Grignon ", Bibliothèque et Archives nationales du Québec, [En ligne], [http://www.banq.qc.ca/histoire_quebec/parcours_thematiques/ ClaudeHenriGrignon/chg_index.jsp] (25 mai 2012).

${ }^{36}$ Une admiration et une amitié qui sont clairement exprimées dans les mémoires posthumes de Claude-Henri Grignon, Olivar Asselin, le pamphlétaire maudit, sous la direction de Pierre Grignon, Trois Pistoles, Éditions Trois-Pistoles, 2007. 
de la publication de son roman Trente Arpents en 1938) avaient publié une anthologie composée de satires impitoyables consacrées aux auteurs parmi les plus célèbres de l'époque. Ainsi passèrent au miroir déformant les Henri Bourassa, Camille Roy, Lionel Groulx, Henri Letondal, Valdombre (pseudonyme sous lequel Claude-Henri Grignon a déjà entamé une polémique soutenue avec Victor Barbeau, auteur de sa propre série de charges littéraires, Têtes de Turc) et bien d'autres. La parodie de Valdombre s'intitule "Un porc: Léon Bloy ", dont ces passages donnent bien le ton :

[...] je me crois en droit de parler sans fausse rhétorique de l'être le plus putride qu'un Pégase en rut ait jamais vomi sur le plancher croulant des lettres françaises.

[...] Je n'écris ni pour les imbéciles, ni pour les gens intelligents. J'abandonne avec joie les sous-Barbeau et les sous-Montpetit à leurs appétits de conférences littéraires et de pâtisserie française. Je m'adresse à moi seul, c'est-à-dire à personne. Qu'on se le tienne pour dit. [...] je trouve belle l'attitude de Celui qui s'érige, dans le Désert, en dépuratif de la Langue canadienne outragée.

Quand on a clamé une fois de telles vérités, il ne reste plus qu’à gravir le calvaire - quel calvaire! - formé de l'amoncellement des calomnies, des crachats et des cadavres morts-nés [sic] des Nigoguistes d'autrefois. [...] il n'est personne, je crois, à part, bien entendu, les suaves monomanes de la Clarté, et après le très indulgent exposé qui précède, qui ne conclue à l'assimilation parfaite de Léon Bloy avec le dernier porcin de la plus lamentable race ${ }^{37}$.

Cette désapprobation pseudo-valdombrienne du Nigog, mais aussi des efforts menés par Édouard Montpetit et Victor Barbeau pour la défense d'une langue française de qualité, est ici réitérée dans un langage excessif caractéristiquement bloyen, plein de vomissements, de crachats, de putréfaction, de mort.

37 "Un porc : Léon Bloy », dans Francoeur et Panneton, Littératures... à la manière de..., p. 25-26. 
Un an plus tard, Valdombre est invité à écrire un texte pour le volume publié par l'École littéraire de Montréal à l'occasion de son $30^{\mathrm{e}}$ anniversaire. Il en profite pour composer un hommage (qui n'est pas parodique, cette fois) à Léon Bloy ${ }^{38}$. En 1931, Germain Beaulieu tourne en légère dérision les efforts de l'École, dont il est membre, dans le petit ouvrage Nos Immortels, publié aux Éditions Albert Lévesque. Illustré par des caricatures signées Albéric Bourgeois, le volume poursuit dans la veine établie par Francoeur et Panneton, Beaulieu et ses contemporains étant eux-mêmes ciblés. Grignon se penche sur le phénomène de ces portraits pleins d'esprit dans un texte qu'il reprend en 1934 dans son recueil Ombres et clameurs : regards sur la littérature canadienne. Il se révèle assez pessimiste visà-vis de l'état de la critique contemporaine :

Ces fameuses lettres canadiennes, on les a bien mêlées depuis une dizaine d'années. Des critiques distingués, autorisés, mielleux, emmiellants, parfois frondeurs, chatouilleux, envieux, dogmatiques, enténébrés, invertébrés et ignorants ont écrit sur les livres de chez nous des choses inouïes, ridicules, professorales et encombrantes. M. Beaulieu, au contraire de ces pontifes, ne pose pas au critique : mais son livre est peut-être jusqu'à présent la meilleure analyse qui ait encore paru sur les œuvres des littérateurs canadiens $[\ldots]^{39}$.

Trente-quatre ans plus tard, Grignon évoque l'impact de cet écrit :

D'abord dans les Soirées de l'École Littéraire où mon article fulgurant fit l'effet d'une bombe dans une mare aux grenouilles. Plus tard dans des journaux tels que La Minerve d'Arthur Sauvé, le Canada, L'Ordre, la Renaissance de l'incomparable polémiste Olivar Asselin qui adorait Léon Bloy et se paya le luxe de lui rendre visite à Bourg-la-Reine, le 30 juin 1917. Croyez-le ou non,

38 Les soirées de l'École littéraire de Montréal : proses et vers / par Englebert Gallèze, Valdombre, J.-A. Lapointe, Albert Laberge, Albert Ferland, Albert Dreux, Germain Beaulieu, Damase Potvin, Ubald Paquin, Louis-Joseph Doucet, Alphonse Beauregard, Jules Tremblay, G. A. Dumont, W.-A. Baker, Albert Boisjoly, Montréal, Beauceville, L'Éclaireur, 1925.

39 Claude-Henri Grignon, "Germain Beaulieu », dans Ombres et clameurs : regards sur la littérature canadienne, Montréal, Éditions Albert Lévesque, 1933, p. 148-149. 
je ne manquai pas l'occasion de parler de Bloy, presque toutes les semaines pendant trois ans, dans la feuille En Avant de T.-D. Bouchard, lequel politicien ne fut jamais que je sache un Père de l'Église. Mais Bouchard plaçait le respect de la liberté de parole, la liberté de croire et de penser au-dessus de tout ${ }^{40}$.

C'est au cours des années 1930, dans l'amitié profonde qui se tissa entre Claude-Henri Grignon et Olivar Asselin, que l'on voit le poids qu'exerce Léon Bloy - force souterraine qui témoigne de l'importance croissante de la parodie et de l'ironie dans l'ensemble de la culture polémique au Québec - sur leur imaginaire. Asselin a quelques conseils pour son ami, qui est alors en rédaction de son célèbre roman Un homme et son péché. Non la moindre des contributions de Grignon est sa création d'un monde gouverné par l'archétype du riche misérable, tout en ayant recours à un idiome canadien-français qui jura avec les préceptes de l'ordre linguistique alors courants. L'impact de ce récit se mesure non seulement à l'échelle de ses incontestables qualités de langue et d'expression, mais aussi par la création d'une variante tragique du modèle de l'Habitant transformé ainsi en anti-héros monumental. Or nous savons maintenant qu'en ce qui a trait à la sexualité dans le roman, Asselin aurait souhaité que Grignon accentue davantage la brutalité, notamment dans l'épisode du viol de Donalda par Séraphin ${ }^{41}$. Lorsque Grignon doit accepter de publier une édition expurgée, il reconnaît avec Asselin que tous deux ont trahi, à un moment ou à un autre, l'esprit de Bloy : Bloy, l'incorruptible, voué à la plus abjecte pauvreté; Bloy, mystique, dont toute l'écriture était une embardée contre l'ennemi. Or les récentes

40 Sœur Marie Saint-Louis de Gonzague, Léon Bloy face à la critique, thèse de doctorat (lettres), Québec, Université Laval, 1958.

${ }^{41}$ Grignon se penche sur l'importance de Bloy pour Asselin dans la biographie de son ami qu’il rédige dans les années 1960 : Grignon, Olivar Asselin, le pamphlétaire maudit. Les extraits qui nous intéressent ont cependant été reproduits dans l'appendice III de l'édition critique d'Un homme et son péché, sous la direction d'Antoine Sirois et Yvette Francoli, Montréal, Presses de l'Université de Montréal, 1986 [1933], p. 226-235. Ces extraits montrent que Grignon et Asselin voient en Bloy l'exemple du critique sans merci et incorruptible. 
études bloyennes ont vu dans cette " écriture de la douleur " une satire sociale et littéraire qui est absolue : en cela, Bloy s'inscrirait dans la suite de la conception préconisée par Charles Baudelaire, pour qui le rire satanique rejoint le grotesque dans le régime du comique absolu ${ }^{42}$.

Grignon lui-même établit clairement le lien entre Bloy et Baudelaire dans une notice critique qu'il publie en 1934 au sujet de l'écrivain Harry Bernard (1898-1979), qu'il accuse d'avoir mal compris le poète - maudit s'il en est un - Charles Baudelaire (18211867). Grignon exalte ce qu'il conçoit comme étant une réalisation profondément catholique : une poésie consacrée à la déchéance la plus totale, poésie qui pose ainsi la question de l'existence de Dieu et de Sa grâce. La cible ultime dans la représentation ordonnée de la société était, paradoxalement, l'Église catholique elle-même, ce que reconnaît bien Grignon dans son essai Ombres et clameurs : regards sur la littérature canadienne:

C'est toujours la même question torturante et difficile qui se dresse devant nous : celle du catholicisme et du mysticisme, sujet lourd de conséquences morales que peu de cerveaux sont capables de résoudre. Personnellement, j'accepte, je comprends, je saisis le catholicisme tel qu'adoré, tel que pratiqué, tel qu'entendu, tel qu'enseigné par Baudelaire, Verlaine, Rimbaud, Huysmans, D’Aurevilly, Villiers de l'Isle Adam, Léon Bloy et Charles Péguy.

Baudelaire reste le vrai catholique qui possède l'amour de Dieu plutôt que la crainte de l'enfer. Il ne joue pas à la confession, ni à la pénitence. Il est sincère, et un malaise continuel le tient cloué sur la croix aux pieds du Christ. Et parce qu'il est sincère il dit des vérités profondes qui déchirent les entrailles des faux catholiques, des catholiques en sucre d'orge, comme il en est malheureusement trop. De là l'anathème qui pèse sur l'œuvre de Baudelaire ${ }^{43}$.

${ }^{42}$ Voir Royer et Sarrazin, Léon Bloy, t. 2 : Le rire de Léon Bloy, p. 146. Sur le comique de Baudelaire, voir Michèle Hannoosh, Baudelaire and Caricature: From the Comic to an Art of Modernity, University Park, The Pennsylvania University Press, 1992.

${ }^{43}$ Grignon, Ombres et clameurs, p.185. Au sujet de Baudelaire et de la métaphore de la charogne, voir Ainslie Armstrong Mclees, Baudelaire's "Argot Plastique”: Poetic 
Asselin et Grignon : deux versants, peut-être pas si éloignés l'un de l'autre, d'une réflexion remarquable qu'ils portent sur une composante assez troublée de la culture française et de la renaissance nationale. Cette composante concerne sans doute la relation de l'individu à Dieu, mais c'est une relation telle qu'elle peut être vécue "à haute voix ", dans la culture littéraire et artistique. Les façons de vivre cette relation ouvertement sont en mutation dans la société des années 1930, et les manières de la concilier avec le devoir public ne sont pas aisément données ni vécues n’importe comment. Avec L'Ordre, qu'il ferme avec l'édition du 11 mai 1935 lorsque le journal est frappé d'interdiction par l'évêque de Montréal, et son successeur éphémère La Renaissance, la carrière d'Asselin journaliste tire à sa fin. Suite à un bref emploi pour le gouvernement, il se consacre dans ses derniers mois de vie à L'CEuvre de la merci, mourant en ascète en avril 1937, à peu près vingt ans après sa rencontre avec Léon Bloy ${ }^{44}$. Son ami Claude-Henri Grignon fera d'Olivar Asselin et de Léon Bloy les deux pierres de touche des Pamphlets de Valdombre qu'il rédige et publie de décembre 1936 à mai 1943. Dès le deuxième numéro (janvier 1937), Grignon oppose Léon Bloy à Maurice Duplessis dans le texte "Le Christ à Québec" (" contre Maurice Duplessis dans Trois-Rivières, il aurait perdu son dépôt $t^{45} »$ ). En fait, Bloy figure largement dans le cadre référentiel de Grignon au travers des Pamphlets. Grignon lui consacre un numéro complet (« Bataille autour de Léon Bloy ", mai 1938) et il en fait le sujet direct ou indirect d'une quinzaine de textes individuels. S'étonnant constamment que l'exemple de Bloy ne soit pas placé au cœur du programme des écoles de la province - on imagine l'effet sur la population scolaire! - Grignon réussit tout de même à répandre la " bonne nouvelle " bloyenne, si bien qu'en

Caricature and Modernism, Athens (É.-U.) et Londres, University of Georgia Press, 1989.

44 Marcel-Aimé Gagnon, Olivar Asselin toujours vivant, Montréal, Presses de l'Université du Québec, 1974, p. 69.

45 Claude-Henri Grignon, "Le Christ à Québec ", Les pamphlets de Valdombre, vol. 1, $\mathrm{n}^{\circ} 2$ (janvier 1937), p. 55-68. 
1941 il est en mesure de constater les résultats de sa propagande lorsque le révérend père Gustave Lamarche et le critique littéraire du journal Le Droit, Guy Sylvestre, commencent à s'y intéresser ${ }^{46}$. Entretemps, Grignon honore son regretté ami Asselin en lui consacrant à son tour un numéro entier dans lequel il accusera Hermas Bastien, premier biographe d'Asselin, d'avoir largement plagié ce dernier ${ }^{47}$. Jusqu'à la dernière livraison des Pamphlets, Grignon ne cesse d'invoquer les exemples d'Asselin et de Bloy, dont on peut imaginer qu'il contribue à faire circuler les noms auprès d'un lectorat pour lequel il publie, à partir de 1940, des extraits du scénario de son feuilleton radiophonique Les Belles Histoires des pays d'en haut dans les livraisons successives des Pamphlets. Ces extraits en deviennent d'ailleurs, de plus en plus, le contenu principal. Quelque part dans l'imaginaire du Québec des années 1940-1943, les personnages issus de l'univers d'Un homme et son péché accèdent à la postérité qu'on connaît, accompagnés d'un éditeur canadien regretté et d'un écrivain français catholique dont Grignon partage les valeurs et les stratégies polémiques farouches.

\section{Postérité de Bloy au Québec, 1943-1960}

Après la Seconde Guerre mondiale, la présence de Bloy dans le paysage canadien-français est assurée par des figures actives dans les milieux culturel, religieux et politique, qui sont d'une remarquable diversité. Éric Walbecq nous montre précisément le cheminement

46 Claude-Henri Grignon, «Au Pays de Québec : le R. P. Gustave Lamarche devant Léon Bloy ", Les pamphlets de Valdombre, vol. 4, n 3 (août 1940), p. 14-121; "Poisons distillés : un scandale épouvantable ", Les pamphlets de Valdombre, vol. 4, $\mathrm{n}^{\circ} 10$ (mars 1941), p. 332-336. Dans ce texte, Grignon réfere aussi à une notice publiée dans Le Quartier latin du 17 janvier 1941 et rédigée par Guy Genest, qui est coupable, selon Grignon, d'une " prose aussi primaire que malpropre sur Léon Bloy ".

47 «Au Pays de Québec: Olivar Asselin et les polémiques d'autrefois », Les pamphlets de Valdombre, vol. 2, n 11 (octobre 1937), p. 447-473. Grignon s'en prend à l'ouvrage de Bastien (Olivar Asselin, Montréal, Bernard Valiquette, 1938) dans "Au Pays de Québec : Asselin vivant ", Les pamphlets de Valdombre, vol. 2, n 5 (avril 1938), p. 220-231. 
des structures associatives qui entretiennent le flambeau bloyen jusqu'aux lendemains de la Révolution tranquille. Revenons sur certains éléments. L'étudiant Raymond Barbeau (1930-1992), fondateur de l'Alliance laurentienne (précurseur du Rassemblement pour l'indépendance nationale), y consacre la thèse qu'il soutient à la Sorbonne en $1955^{48}$. À la même époque, sœur Marie de Gonzague en fait une étude approfondie, qu'elle fera paraître en 1959 avec une préface signée par Grignon ${ }^{49}$. Dans un autre recoin de la vie culturelle, on peut signaler, entre de nombreux autres exemples, le nom de Guy Robert, futur directeur du Musée d'art contemporain, qui signe des billets critiques au sujet de Bloy au tournant des années $1960^{50}$. Enfin, l'influence qu'a pu exercer Léon Bloy sur les milieux culturels du Québec, au moment où la société s’apprête à basculer de manière définitive vers un nouveau régime politique et symbolique, est attestée par le père jésuite Guy Courteau dans le numéro de janvier 1959 de la Revue d'histoire de l'Amérique française dirigée par Lionel Groulx ${ }^{51}$. Cette étude bibliographique en dit long sur la place réservée à Bloy dans l'imaginaire de certains penseurs et écrivains qui le rattachent, comme Raymond Barbeau, au rêve d'un Canada français indépendant que serait cette mythique Laurentie. Le père Courteau voit cette influence se dessiner en quatre époques :

la première, de 1878 à 1905 , ce sont les lueurs nocturnes ou lointaines de l'aube; la deuxième, de 1906 à 1931, les signes avant-coureurs de gloire ou l'aurore (les premiers bloyens : Fournier, Asselin, Valdombre, préparent l'infiltration de Bloy en notre Laurentie); la troisième, de 1933 à 1948, Valdombre et ses satellites ou les grands semeurs des matins de gloire bloyenne; la quatrième, de 1949 à 1957, espoirs de profonde pénétration bloyenne; $\ldots$ ou le plein jour se lève ${ }^{52}$.

48 Publié deux ans plus tard sous le titre Un prophète luciférien, Léon Bloy (essai), Paris, Aubier, Montaigne, 1957.

49 Sœur de Gonzague, Léon Bloy face à la critique.

50 Revue dominicaine, novembre 1957, p. 251; Le Petit Journal, 12 août 1962, p. 10.

51 Guy Courteau, s.j., "Léon Bloy en Amérique française ", Revue d'histoire de l'Amérique française, vol. 13, $\mathrm{n}^{\circ} 1$ (juin 1959), p. 79-92. Une étude comparable pour les années 1959-2012 serait sans doute à faire.

52 Ibid., p. 80-81. 
Bien sûr, l'espoir laurentien est ici bien loin de celui qu'hébergeaient les équipes du Devoir et de Vrai, qui étaient proches de la polémique du frère Untel (Jean-Paul Desbiens, 1927-2006). En réponse à la plainte exprimée en septembre 1959 par André Laurendeau sur l'état de la langue française au Québec ${ }^{53}$, Desbiens poursuit un thème autrefois cher à Olivar Asselin. De son nom religieux de frère Pierre Jérôme, Desbiens entretient avec Laurendeau une correspondance qui atteint rapidement des proportions importantes. La suite de l'histoire est bien connue, car les lettres de Desbiens sont éditées par les Éditions de l'Homme sous le titre Les insolences du frère Untel, volume qui fait date dans l'historiographie de la Révolution tranquille. Dans la préface ("L'avertissement », en fait), Desbiens explique la responsabilité dont il s'est chargé :

Déblayer n'est pas œuvrer; il faut pourtant commencer par déblayer; et avant même de déblayer, il faut démolir. Saint Léon Bloy se déclarait entrepreneur en démolition. Je relaye Léon Bloy, génie en moins (il faut que je dise ça, mais je n'en crois rien), à cinquante ans de distance, comme il convient dans un pays où l'on vit avec un retard de deux révolutions et demie : horloge d'Amérique, heure du Moyen-Âge ${ }^{54}$.

C'était en effet d'un entrepreneur en démolition dont le Québec avait alors besoin : démolition d'un homme, d'un monument, d'un mythe. LaPalme et Desbiens vont, chacun à leur façon, répondre au type de désespoir au sujet duquel Georges-Émile Lapalme sera éloquent dans le deuxième tome de ses mémoires, Le vent de l'oubli:

Maurice Duplessis restait l'ennemi paré de tous les défauts, celui qu'il fallait politiquement assassiner. [...] C'est en l'écoutant un soir en chambre que, froidement surpris, je sentis le passage frôlant de l'Histoire. Était-ce possible? En scrutant les galeries bondées,

53 Candide (pseudonyme d'André Laurendeau), "La langue que nous parlons ", Le Devoir, 21 octobre 1959, p. 4.

${ }_{44}$ Jean-Paul Desbiens, Les insolences du frère Untel, Montréal, Éditions de l'Homme, 2000 [1960], p. 18. 
je m'interrogeais : Pourrons-nous jamais détruire le mythe vivant qu'il crée de rien? Il est en train d'usurper un rôle à la Mercier. Le pire, c'est qu'il réussit ${ }^{55}$.

Peu à peu, je m'étais aperçu qu'il n'y avait pas eu hallucination collective et que Maurice Duplessis avait entendu d'avance, comme s'il eut plaqué un stéthoscope, les vibrations populaires. Je le voyais développer une stature différente, enveloppée dans ce qui n'était plus que le voile diaphane d'un mythe. Il prenait corps avec un certain rêve canadien-français chargé de trop de passé, pas assez d'avenir, s'ouvrant tout à coup sur une réalité lointaine mais possible. Je le voyais autrement. Je le voyais invincible : j'ai lâché le mot qui m'explique ${ }^{56}$.

Le malheureux chef des libéraux ne savait que trop bien à quel point l'imaginaire de son temps s'était empreint de ces formes symboliques. Si Duplessis relevait déjà du mythe, Lapalme lui-même devait bien relever de quelque chose, lui aussi. Il ne s'épargne pas non plus, car il se souvient comment Duplessis « ... eut un jour un mot délicieux. Pendant une conférence de presse un journaliste lui avait posé cette question : "Que pensez-vous de Lapalme?" - "Lequel ? Le caricaturiste ou la caricature?"57"

Il faudra sans doute revenir en détail sur la place de Léon Bloy dans cet "imaginaire de la fin " (des temps de Duplessis). Au-delà des quelconques figures mythiques ou caricaturales, c'est l'ordre des choses - le lieu même de l'entente - entre l'État et l'Église que la verve apocalyptique de Bloy nous semble travailler, ronger et déranger. On imagine que la lecture de Bloy dans les années 1950 servait surtout à repenser la province de Québec comme un territoire qui, tout en résistant aux excès sécularistes de la Troisième République, n’en fut pas moins lâchement livré à un consensus bourgeois, où la foi devient

55 Georges-Émile Lapalme, Le bruit des choses réveillées : mémoires, t. 2 : Le vent de l'oubli, Outremont, Leméac, 1970, coll. «Vies et mémoires ", p. 150.

56 Ibid., p. 181.

${ }^{57}$ Georges-Émile Lapalme, Le bruit des choses réveillées, p. 115. 
routine et où le rêve d'une vie collective autrement imaginée est toujours mort-né. En attendant ce travail plus exhaustif, l'image et la conjoncture que nous avons souhaité mettre en lumière ici peuvent se préciser, au terme de cette étude, autour des deux composantes de l'image : l'homme de pouvoir, le mythe et sa caricature, certes, mais aussi son territoire. Ainsi, c'est non seulement sur la figure dénudée du grand Maurice qu'il nous fallait porter notre attention; nous devions aussi réfléchir sur les signes de l'espace qu'évoque le dessin de Robert LaPalme.

\section{Fin de parcours : le grand Maurice, un errant sur la terre de Caïn}

"Ta peur de Pierre, Jean, Jacques te fait faire des embardées » - lisons, si l'on veut, ta peur de tout un chacun, ou bien encore de Pierre [Desmarais], Jean [Drapeau], Jacques [Hébert] -, « pour te défendre tu attaques comme un désespéré " (nous soulignons). Si donc nous nous permettons de suivre cette allusion au Désespéré de Léon Bloy, c'est à cause d'une coïncidence qui provoque une réflexion sur des échos, et non sur des liens de causalité. Quelle méthode! En histoire de l'art, de même qu'en études littéraires ou en histoire (de la) politique, rarement se permet-on de contourner la patiente et obligatoire construction d'une relation causale avant de justifier une telle comparaison, par ailleurs profondément anachronique. Image de 1958 au Québec, roman de 1887 en France : la distance est si vaste. Comment la justifier?

Pourtant, on pourrait reprendre, dans cette démarche d'histoire expérimentale, la méthode employée par les organisateurs de l'exposition Hitchcock et l'art, présentée en 2000-2001 au Musée des beaux-arts de Montréal et au Centre Pompidou à Paris. L'exposition avait relevé le pari d'une mise en relation d'images foncièrement dissociées, sauf par leur participation à un régime d'échos et de ressemblances, comme autant de symptômes d'une même culture (culture de crises névralgiques, dirait-on). On pouvait alors présenter les écrits et les images des $\mathrm{XIX}^{\mathrm{e}}$ et $\mathrm{XX}^{\mathrm{e}}$ siècles anglais, français et 
américain non pas comme sources directes de l'iconographie dont Alfred Hitchcock meublait ses films, mais plutôt comme indices des préoccupations (ou obsessions, si l'on veut) communes et transhistoriques qui marquent une grande temporalité ${ }^{58}$. Dérogation au devoir du bon "chronologue ", sans doute, mais aussi regard anamorphique qui permet d'échapper au présentisme constamment réincarné d'une culture qu'il faudrait considérer plutôt par époque, en s'appropriant une méthode presque anthropologique d'analyse de l'image et du discours. Dans le cas de Robert LaPalme, dont le métier (et sans doute aussi la conviction) était, depuis longtemps, d'être obsédé par le corps de Duplessis qui refusait sa mort, tout autant que par le régime politique qui opposait le même refus, comment la violence de son image peut-elle prendre son plein sens, comment peut-elle traverser la brèche qu'elle creuse dans l'espace de la une de Vrai?

Pour y parvenir, nous avons choisi de nous consacrer à l'image, en la comparant avec un texte qui ne lui appartient pas et au pari d'un rapprochement qui relève davantage d'Aby Warburg (18661929). Nous avons constaté qu'on ne peut découper les images pour les réunir sur la base de ressemblances, comme tentait de le faire Warburg dans son projet Mnémosyne, mais qu'il faut plutôt les associer selon la logique de l'exposition - volontairement anachronique consacrée à Hitchcock et l'art ${ }^{59}$.

La bouche ouverte, la langue sauvage, féroce, ce Duplessis à la nudité grotesque semble répondre à quelques-unes des premières phrases du roman de Bloy : "Ah! le couteau eût mieux valu, sans doute, le rudimentaire couteau du chourineur filial [...] Vous m'avez vu [...] coiffé d'une ordure cylindrique, dénué de vêtements, de

58 Dominique Païni et Guy Cogeval (dir.), Hitchcock et l'art, coïncidences fatales, Milano, G. Mazotta; Montréal, Musée des beaux-arts de Montréal, 2000.

59 Le Bilderatlas Mnemosyne (1927-1929) d'Aby Warburg, atlas d'images organisées selon des principes foncièrement anachroniques, est conservé au Warburg Institute à Londres. Pour une récente analyse exhaustive de la méthode expérimentale de Warburg, voir Georges Didi-Huberman, L'image survivante : histoire de l'art et temps des fantômes selon Aby Warburg, Paris, Les Éditions de Minuit, 2002, coll. " Paradoxe». 


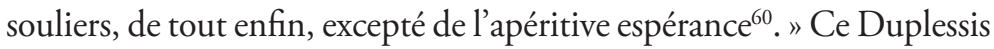
ne reprend pas au pied de la lettre le rôle de Marie-Joseph Caïn Marchenoir, dont le parricide ouvre Le désespéré. En fait, il ne pourrait être, en réalité, plus éloigné du journaliste, du dandy, de l'ascète, du mystique obsédé par l'apocalypse qu'ont été et Léon Bloy et l'avatar qu'il crée pour se représenter dans ce roman. Le texte de Bloy est profondément imbu d'une forme de passion satirique, par laquelle la signification d'une autre Passion, au sens religieux, s'oppose constamment au lecteur tout en le confrontant, en tant que signe de la Révélation, et donc de la fin apocalyptique des temps. Bloy et Marchenoir s'insurgent contre l'Église qui s'est vendue à la société capitaliste du XIX ${ }^{e}$ siècle. Dans cette logique, le régime de l'Église est le régime de la temporalité humaine; elle est consécutive à la faute originelle et doit être rachetée afin de préparer la Rédemption. Tout ceci semble bien loin de Duplessis! Sauf dans la mesure où le concordat entre l'Église et le pouvoir temporel au Québec se trouve précisément au cour des préoccupations qui vont alimenter la Révolution tranquille. Dans ce contexte, l'association de Duplessis à la figure de Caïn - et à son errance - devient riche de sens. Le premier ministre s'était notamment défini en matière de développement économique pour le Québec par ses politiques d'exploitation de ressources naturelles au territoire de l'Ungava, la soi-disant terre de Caïn du Québec. Tuant Abel, Caïn le détruit, mais il chasse Dieu aussi. Dans une perspective bloyenne, ce dessin pourrait être vu comme une ultime accusation à l'endroit de Maurice Duplessis contre la constante manipulation de l'Église catholique au nom d'un concordat qui livre les ressources naturelles de la province de Québec aux étrangers (aux Américains, notamment); concordat qui, dans la perspective bloyenne, finit paradoxalement par chasser Dieu de l'ordre temporel et de l'espace du peuple. Or, comme l'ont montré Jean-Philippe Warren et E. Martin Meunier, un mouvement important est à l'œuvre dans ces années, et ce, à l'intérieur même de l'Église, pour démonter l'alliance entre le cléricalisme et l'État, réforme qui doit aboutir au

${ }^{60}$ Léon Bloy, Le désespéré, Paris, Mercure de France, 1933, p. 9. 
transfert des responsabilités sociales (notamment en ce qui concerne l'organisation des services de santé et d'éducation), jusqu'alors réservées aux religieux, vers la population dans son ensemble ${ }^{61}$. En même temps, la figure de Duplessis ne deviendrait-elle pas expiatoire, emblématique de la déchéance et de la faute humaine venant racheter d'avance le corps social du Québec? Ce serait trop beau, et la question serait trop parfaite; elle voudrait trop ardemment qu'un lien se dessine (pour ainsi dire) clairement entre les deux éléments de la comparaison. Pour le moment, et pour continuer, il est possible d'admettre, à l'aide des documents et des auteurs que nous avons cités à des fins bien diverses, que la critique bloyenne figure dans les milieux où se prépare le renouveau qui, croit-on et espère-t-on alors, va venir au Québec. On se croirait au printemps « érable » de 2012.

\section{Conclusion : autour d'une trace fugitive}

Reconsidérer ici le dessin de Robert LaPalme du 22 février 1958 offrait l'occasion de le constituer comme trace, si indirecte soit-elle, de deux présences dans l'histoire des discours polémiques au Québec. Trace fugitive, incertaine, dira-t-on, d'une première influence, difficile à saisir - celle de Léon Bloy - et d'une deuxième, celle du dessin satirique dans l'espace discursif. En effet, au terme de cette relecture, est-il possible d'affirmer que cette trace prend son sens parmi l'ensemble des traces bloyennes qui ont été exhumées et placées dans le présent texte? S'il y a leçon à tirer, ou projet à cerner, il faudrait surtout se pencher sur la latence comme phénomène caractéristique du transfert des comportements culturels les plus violents dans la société canadienne-française. Latence assez remarquable qui semble rendre visible de manière bien ambiguë ces traces, du moins dans la perspective du transfert culturel dont on voudrait ici montrer le mécanisme. Les aléas et les contingences de la caricature et de la satire graphique au Québec dans la première

${ }^{61}$ Jean-Philippe Warren et E. Martin Meunier, Sortir de la grande noirceur : l'horizon personnaliste de la Révolution tranquille, Sillery, Septentrion, 2002. 
moitié du Xx $x^{\mathrm{e}}$ siècle nous montrent toute l'importance d'une étude attentive de certains milieux de l'élite qui façonnaient, souvent de manière souterraine et à l'aide d'une dissidence résiliente, de nouvelles perspectives culturelles. L'absorption, par le Québec, d'une vision anticléricale qui partait, paradoxalement, de l'intérieur du catholicisme en tant que tel, et de l'intérieur des espaces du journalisme, se fait en marge des mécanismes revendiqués par l'Église depuis l'encyclique Rerum novarum $(1891)^{62}$. Catholique dévot qui exigea cependant l'autonomie de l'éducation publique par rapport à l'Église, nationaliste fervent qui se fit apôtre du meilleur de la civilisation française, Olivar Asselin était lui aussi de cette génération qui comprit l'importance de la presse écrite au sein de laquelle il plaça, au premier rang, les stratégies ironiques et satiriques - celles-ci étant autant visuelles que textuelles. Sa pleine ferveur bloyenne, cependant, ne put se transférer dans les discours publics qu'à la mesure des limites de ceux-ci. Les témoignages d'un Claude-Henri Grignon, notamment, nous permettent de croire à une circulation privée plus retentissante. Les réseaux de sociabilité canadiens-français fournissent aussi une structure de diffusion des idées et des figures bloyennes, notamment après la Seconde Guerre dont l'horreur ne pouvait que faire écho aux visions du Pèlerin de l'Absolu lui-même. C'est peut-être dans l'espace créé par cet écho que Léon Bloy put refaire surface pour participer à la fois aux parachèvements, entrepris notamment par Robert LaPalme et le frère Untel, de l'ordre des représentations par lesquelles cette société se reconnaissait, ainsi qu’à l'enfantement des nouvelles représentations idéologiques et

${ }^{62}$ Avec Rerum novarum, le pape Léon XIII avait signalé l'importance pour l'Église de se tourner vers une action sociale et de maîtriser, justement, les infrastructures de la représentation sociale, entre autres, celles du journalisme d'information et polémique - leçon bien comprise au Québec avec la fondation de l'Action catholique (1907) et du Devoir (1910). Pour une perspective plus générale portant sur le Vatican et les préoccupations sociales du tournant à la fin du XIx ${ }^{\mathrm{e}}$ siècle, voir Yvan Lamonde, Histoire sociale des idées au Québec, t. 1 : 1760-1896, Montréal, Fides, 2000, p. 435-464. 
médiatiques qui ont si longtemps permis à la notion de Révolution tranquille de se coller à la narration de la société québécoise, qui s’est pensée en devenir depuis 1960.

Voilà bien des pistes, qui mènent surtout vers la littérature et le langage : voilà le problème pour l'enquêteur en histoire de l'art qui cherche à comprendre une image, ou encore la création et l'évolution de toute une iconographie. Mais justement, entreprendre une telle enquête sur le Québec des décennies 1930, 1940, 1950, où l'on s'interroge sur les courants de pensée, c'est aborder non seulement les idéologies, mais aussi (et surtout, puisqu'il est aisé de mener ainsi une réflexion simultanée sur le texte et l'image, l'étude de l'idéologie ne se limitant pas à l'étude des discours dits et écrits) ces traversées de l'imaginaire. C'est par ce chemin qu'on peut tenter de caractériser une image comme Un valentin au grand Maurice et la réintégrer au plus large éventail des discours émanant de et portant sur la politique québécoise, tels que ces discours prennent forme, en 1958, autour de la figure redoutée de Maurice Duplessis, figure dont Georges-Émile Lapalme a bien montré comment elle a largement occupé l'imaginaire de l'époque. Refaire un tel parcours à la recherche de traces, c'est se dire, à nouveau, que l'image, la représentation politique, l'inventivité stylistique, participaient à la constitution des discours, et ce, autrement ou pas seulement à partir des iconographies issues des réseaux des beaux-arts. La question de l'image dépasse la force que pouvaient avoir les productions des automatistes, des plasticiens et du plein milieu des avant-gardes, force que l'histoire de l'art s'est nécessairement empressée de caractériser comme étant à l'avant-plan des discours qui menaient le Québec vers la Révolution tranquille. Comme on le voit avec l'image de Robert LaPalme du 22 février 1958, le régime des représentations visuelles devait permettre de confronter le corps, même si ce n'était que par les codes de l'ironie et de la satire visuelle selon lesquels est construite la caricature dans la presse à grand tirage.

La réflexion qu'a menée l'historien de la caricature Bertrand Tillier sur la violence dans la représentation satirique du corps peut nous aider à penser la perspective autour de l'image de LaPalme. 
C'est une perspective grâce à laquelle on peut entendre l'écho, un peu lointain mais pas encore disparu, de la Troisième République. La violence polémique de cette époque atteint sa destination québécoise, car elle avait été préparée par le travail personnel d'Asselin et de Grignon sur Léon Bloy :

Le corps et le visage deviennent, en écho à la personnalisation extrême du pouvoir, les supports symboliques et iconographiques des multiples accusations et dénonciations que prodigue la caricature. Le corps devient un lieu de fracture physique, morale et politique, avec le but de déconsidérer l'individu représenté, son parti ou le groupe auquel il appartient. En exposant le corps et les traits des victimes, les caricaturistes opèrent un amalgame entre les caractères privés et publics, permettant ainsi de multiplier les attaques sur tous les fronts [...] Dans la charge comme dans l'insulte, le corps occupe une place fondamentale. [L'opposition à la caricature] souligne le caractère grossier, vulgaire, obscène, pornographique, scatologique et insultant de ces images. [Les mécanismes langagiers et expressifs de la caricature] procèdent de la critique comme injure. [...] Les linguistes ont depuis longtemps analysé l'hyperprésence du corps et de ses fonctions physiologiques dans l'injure et l'insulte, celui-ci étant le siège unique de toutes nos sensations et de notre affectivité ${ }^{63}$.

On se rappellera que quatre élections successives et des centaines de textes éditoriaux et de caricatures n'avaient pu dérober le pouvoir à Maurice Duplessis. Aujourd'hui, faire un retour sur l'image de LaPalme et sur la dimension bloyenne qu'on lui prête volontiers enquête qui nous mène à interroger les lieux de transfert de la voix acharnée de l'auteur français - nous offre l'occasion de creuser davantage la symbolique que confère la notion de désespoir, en y voyant une manière de rendre visible la nécessité de s'attaquer, si possible, à Duplessis dans son " hyperprésence ». Elle affirme une violence qui se voudrait aussi hyperprésente, pour contrer non seulement Duplessis - en le montrant en tant qu'acteur d'une violence primordiale, "pré-historique ", et témoignant de sa

63 Bertrand Tillier, La RépubliCature : la caricature politique en France, 1870-1914, Paris, CNRS éditions, 1997, p. 73. 
domination sur les discours, que ce soit à l'Assemblée nationale, dans la presse écrite, sur les ondes ou encore dans les rangs et les comtés de sa province -, mais aussi l'impuissance constante du discours qui opposa la classe politique constituée par Le Devoir, Vrai, les André Laurendeau, Gérard Filion, Jacques Hébert et bien d'autres face à la résistance inouïe que leur opposait le quasi invincible Cheuf de l'Union nationale. 